\title{
DERIVATIVE POLYNOMIALS, EULER POLYNOMIALS, AND ASSOCIATED INTEGER SEQUENCES
}

\author{
Michael E. HOFFMAN \\ Mathematics Department \\ U.S. Naval Academy \\ Annapolis, MD 21402 \\ meh@nadn.navy.mil \\ Submitted: February 16, 1999; Accepted: April 2, 1999
}

\begin{abstract}
Let $P_{n}$ and $Q_{n}$ be the polynomials obtained by repeated differentiation of the tangent and secant functions respectively. From the exponential generating functions of these polynomials we develop relations among their values, which are then applied to various numerical sequences which occur as values of the $P_{n}$ and $Q_{n}$. For example, $P_{n}(0)$ and $Q_{n}(0)$ are respectively the $n$th tangent and secant numbers, while $P_{n}(0)+Q_{n}(0)$ is the $n$th André number. The André numbers, along with the numbers $Q_{n}(1)$ and $P_{n}(1)-Q_{n}(1)$, are the Springer numbers of root systems of types $A_{n}, B_{n}$, and $D_{n}$ respectively, or alternatively (following V. I. Arnol'd) count the number of "snakes" of these types. We prove this for the latter two cases using combinatorial arguments. We relate the values of $P_{n}$ and $Q_{n}$ at $\sqrt{3}$ to certain "generalized Euler and class numbers" of D. Shanks, which have a combinatorial interpretation in terms of 3-signed permutations as defined by R. Ehrenborg and M. A. Readdy. Finally, we express the values of Euler polynomials at any rational argument in terms of $P_{n}$ and $Q_{n}$, and from this deduce formulas for Springer and Shanks numbers in terms of Euler polynomials.
\end{abstract}

1. Introduction. Consider the sequences $P_{n}$ and $Q_{n}$ of "derivative polynomials" defined by

$$
\frac{d^{n}}{d x^{n}} \tan x=P_{n}(\tan x) \quad \text { and } \quad \frac{d^{n}}{d x^{n}} \sec x=Q_{n}(\tan x) \sec x
$$

for integer $n \geq 0$. As shown in [12], their exponential generating functions

$$
P(u, t)=\sum_{n=0}^{\infty} P_{n}(u) \frac{t^{n}}{n !} \quad \text { and } \quad Q(u, t)=\sum_{n=0}^{\infty} Q_{n}(u) \frac{t^{n}}{n !}
$$

are given by the explicit formulas

$$
P(u, t)=\frac{\sin t+u \cos t}{\cos t-u \sin t} \quad \text { and } \quad Q(u, t)=\frac{1}{\cos t-u \sin t} .
$$

1991 Mathematics Subject Classification. 11B83, 11B68, 05A15.

Key words and phrases. Tangent numbers, secant numbers, André numbers, Springer numbers, snakes, generalized Euler and class numbers, Euler polynomials. 
In $\S 2$ we obtain from identities in these generating functions some useful relations among values of the polynomials (Theorem 2.2 below), and recall from [12] a result (Theorem $2.3)$ relating the polynomials to series of reciprocal powers. In $\S 3$ we apply results of $\S 2$ to the computation of $P_{n}(u)$ and $Q_{n}(u)$ for $u=0,1, \sqrt{3}$, and $1 / \sqrt{3}$, in the process obtaining several integer sequences studied by Glaisher $[9,10,11]$.

In $\S 4$ we give a combinatorial interpretation of the values of the derivative polynomials at 0 and 1. These values give the Springer numbers of the irreducible root systems $A_{n}, B_{n}$, and $D_{n}[19]$, which also count the corresponding types of "snakes" as defined in [3]. (Snakes for the root system $A_{n-1}$ are alternating permutations of $\{1,2, \ldots, n\}$, whose study dates back to André [2].) This follows from comparison of equations (1) with the generating functions found in [19], but we also give combinatorial proofs using snakes (Theorems 4.2 and 4.3). Results from $\S 3$ then give identities for the Springer numbers (e.g., Proposition 4.4).

In $\S 5$ we recall the definition of the the "generalized Euler and class numbers" of Shanks [17]. These are arrays of positive integers $c_{a, n}$ and $d_{a, n}$. The first two "rows" (i.e., the $c_{a, n}$ and $d_{a, n}$ with $\left.a=1,2\right)$ are the Springer numbers of the preceding paragraph; we show that the third row of Shanks's numbers are given by the values $P_{2 n}(\sqrt{3})$ and $Q_{2 n-1}(\sqrt{3})$ of the derivative polynomials. We also give a combinatorial interpretation to the numbers $c_{3, n}$ and $d_{3, n}$ in terms of 3 -signed alternating permutations as defined by Ehrenborg and Readdy [8].

In $\S 6$ we consider the Euler polynomials $E_{n}(x)$, defined by

$$
\frac{2 e^{t x}}{e^{t}+1}=\sum_{n=0}^{\infty} E_{n}(x) \frac{t^{n}}{n !}
$$

Euler polynomials appear in many classical results (see Chapter 23 of [1]). In [6], the values of these polynomials at rational arguments were expressed in terms of the Hurwitz zeta function. Here we give explicit formulas for the Euler polynomials at rational arguments in terms of the polynomials $P_{n}$ and $Q_{n}$ (Theorem 6.1), and use them together with the computations of $\S 3$ to find $E_{n}(p / q)$ for $0 \leq p \leq q$ and $q=2,3,4$ and 6 . We also write the Springer and Shanks numbers in terms of values of the Euler polynomials (Theorem 6.2).

2. Derivative polynomials. From the chain rule it follows that the polynomials $P_{n}$ satisfy $P_{0}(u)=u$ and $P_{n+1}(u)=\left(u^{2}+1\right) P_{n}^{\prime}(u), n \geq 0$, and similarly $Q_{0}(u)=1$ and $Q_{n+1}(u)=\left(u^{2}+1\right) Q_{n}^{\prime}(u)+u Q_{n}(u), n \geq 0$. The following result is then clear by induction on $n$.

Theorem 2.1. Let $n \geq 0$. Then $P_{n}(u)$ is a polynomial of degree $n+1$ consisting of even powers with positive integral coefficients when $n$ is odd and of odd powers with positive integral coefficients when $n$ is even; and $Q_{n}(u)$ is a polynomial of degree $n$ consisting of even powers with positive integral coefficients when $n$ is even and of odd powers with positive integral coefficients when $n$ is odd.

In particular, for $n \geq 0$ we have

$$
P_{n}(-u)=(-1)^{n+1} P_{n}(u) \quad \text { and } \quad Q_{n}(-u)=(-1)^{n} Q_{n}(u) .
$$


THE ElECTRONiC JOURNAL of COMBinAtorics 6 (1999),\#R21

The key properties of the $P_{n}$ and $Q_{n}$ come from two sorts of identities in their corresponding generating functions $P$ and $Q$. First, there are the composition relations

$$
P(P(u, t), s)=P(u, t+s) \quad \text { and } \quad Q(P(u, t), s) Q(u, t)=Q(u, t+s) ;
$$

these follow from the representations

$$
P(u, t)=\tan \left(\tan ^{-1} u+t\right) \quad \text { and } \quad Q(u, t)=\frac{\sec \left(\tan ^{-1} u+t\right)}{\sec \left(\tan ^{-1} u\right)},
$$

equivalent to equations (1) above. Second, there is the functional equation

$$
P(u, t)=P\left(\frac{u^{2}-1}{2 u}, 2 t\right)+\frac{u^{2}+1}{2 u} Q\left(\frac{u^{2}-1}{2 u}, 2 t\right),
$$

which follows from equations (1) and the half-angle formula for tangent (cf. Theorem 3.1 of [12]). The composition relations (3) imply the following relations among values of the polynomials $P_{n}$ and $Q_{n}$.

Theorem 2.2. For nonnegative integers $n$,

$$
\begin{gathered}
P_{n}(P(u, s))-\tan s \sum_{k=0}^{n}\left(\begin{array}{l}
n \\
k
\end{array}\right) P_{k}(P(u, s)) P_{n-k}(u)=P_{n}(u)+\delta_{0 n} \tan s \\
Q_{n}(P(u, s))-\tan s \sum_{k=0}^{n}\left(\begin{array}{l}
n \\
k
\end{array}\right) Q_{k}(P(u, s)) P_{n-k}(u)=(1-u \tan s) Q_{n}(u) .
\end{gathered}
$$

Proof. The composition relation for $P$ gives

$$
P(P(u, s), t)=P(u, s+t)=P(P(u, t), s)=\frac{\sin s+\cos s P(u, t)}{\cos s-\sin s P(u, t)},
$$

or $\cos s P(P(u, s), t)-\sin s P(P(u, s), t) P(u, t)=\sin s+\cos s P(u, t)$. Take the coefficient of $t^{n} / n$ ! and divide by $\cos s$ to get (i). The proof of (ii) proceeds similarly, using the composition relation for $Q$.

In [12] contour integration and expansion into power series were used to obtain closed forms in terms of the $P_{n}$ and $Q_{n}$ for certain series. We need the following definitions. Call a function $\psi: \mathbf{Z} \rightarrow \mathbf{C}$ periodic $\bmod q$ if $\psi(0)=0$ and $\psi(n+q)=\psi(n)$ for all $n \in \mathbf{Z}$, and alternating $\bmod q$ if $\psi(0)=0$ and $\psi(n+q)=-\psi(n)$ for all $n \in \mathbf{Z}$. If $\psi$ is periodic or alternating $\bmod q$, we call it even if $\psi(q-j)=\psi(j)$ for $0<j<q$, and odd if $\psi(q-j)=-\psi(j)$ for $0<j<q$. From [12] we have the following result.

Theorem 2.3. Let $n \geq 0$ be an integer. If $\psi$ is periodic $\bmod q$, then

$$
\sum_{j=1}^{\infty} \frac{\psi(j)}{j^{n+1}}=\frac{\pi^{n+1}}{2 q^{n+1} n !} \sum_{p=1}^{q-1} \psi(p) P_{n}\left(\cot \frac{p \pi}{q}\right)
$$

provided $n$ and $\psi$ have opposite parity. If $\psi$ is alternating $\bmod q$, then

$$
\sum_{j=1}^{\infty} \frac{\psi(j)}{j^{n+1}}=\frac{\pi^{n+1}}{2 q^{n+1} n !} \sum_{p=1}^{q-1} \psi(p) \csc \frac{p \pi}{q} Q_{n}\left(\cot \frac{p \pi}{q}\right)
$$

provided $n$ and $\psi$ have the same parity. 
3. Particular values of derivative polynomials. By setting $u=0$ in equations (1) we see that $P_{n}(0)$ and $Q_{n}(0)$ are respectively the tangent and secant numbers, i.e. the coefficients of $t^{n} / n$ ! in the Maclaurin series of $\tan t$ and $\sec t$. We shall take these numbers as known. They can be computed from the Euler-Bernoulli triangle as discussed in [4] and [3]; see also [13]. In this section we show how to compute $P_{n}(u)$ and $Q_{n}(u)$ for $u=1, \sqrt{3}$, and $1 / \sqrt{3}$ from the tangent and secant numbers.

Set $u=1$ and consider the coefficient of $t^{n} / n$ ! in the functional equation (4) to get $P_{n}(1)$ :

$$
P_{n}(1)=2^{n}\left(P_{n}(0)+Q_{n}(0)\right)= \begin{cases}2^{n} Q_{n}(0), & n \text { even, } \\ 2^{n} P_{n}(0), & n \text { odd }\end{cases}
$$

Now compute $Q_{n}(1)$ using the following result.

Theorem 3.1. For integers $n \geq 0$,

$$
Q_{n}(1)=-\sin \frac{n \pi}{2}+\sum_{2 k \leq n}\left(\begin{array}{c}
n \\
2 k
\end{array}\right)(-1)^{k} P_{n-2 k}(1) .
$$

Proof. Set $u=1$ in Theorem 2.2(ii), and then let $s \rightarrow+\infty i$ so that $\tan s \rightarrow i$ and $P(1, s) \rightarrow i$. This gives

$$
(1-i) Q_{n}(1)=Q_{n}(i)-i \sum_{k=0}^{n}\left(\begin{array}{l}
n \\
k
\end{array}\right) Q_{k}(i) P_{n-k}(1) .
$$

Now $Q(i, t)=e^{i t}$, so $Q_{n}(i)=i^{n}$. Thus, we have

$$
(1-i) Q_{n}(1)=i^{n}-i \sum_{k=0}^{n}\left(\begin{array}{l}
n \\
k
\end{array}\right) i^{k} P_{n-k}(1) .
$$

Take the imaginary part to get the conclusion.

Remarks. 1. This result, together with the corresponding one obtained by taking the real part in the proof, is equivalent to the computation of the $Q_{n}(1)$ from the numbers $P_{n}(1)$ via Seidel matrices as described in [7].

2. Since the $Q_{n}(1)$ turn out to be the Springer numbers $b_{n}$ of the next section, they can be computed via the pair of "boustrophedonic" triangles $L(b)$ and $R(b)$ described in [3] (see also [14]); in fact these triangles are equivalent to Seidel matrices as is explained in [7].

3. The numbers $Q_{n}(1)$ were extensively studied by Glaisher [9,11], who wrote $P_{n}$ for $Q_{2 n}(1)$ and $Q_{n}$ for $Q_{2 n-1}(1)$.

Set $u=-1 / \sqrt{3}$ in equation (4) and examine the coefficient of $t^{n} / n$ ! to get

$$
\left(2^{n}+(-1)^{n}\right) P_{n}\left(\frac{1}{\sqrt{3}}\right)=\frac{2^{n+1}}{\sqrt{3}} Q_{n}\left(\frac{1}{\sqrt{3}}\right) ;
$$

then combine this with the equation obtained by setting $u=\sqrt{3}$ to get

$$
P_{n}(\sqrt{3})=\left(2^{n+1}+(-1)^{n}\right) P_{n}\left(\frac{1}{\sqrt{3}}\right) .
$$

In view of these equations, to find $P_{n}\left(\frac{1}{\sqrt{3}}\right)$ and $Q_{n}\left(\frac{1}{\sqrt{3}}\right)$ it is enough to find $P_{n}(\sqrt{3})$. Our next two results give $P_{n}(\sqrt{3})$ and $Q_{n}(\sqrt{3})$. 
Theorem 3.2. $i$. If $n$ is odd, $P_{n}(\sqrt{3})=\frac{1}{2}\left(3^{n+1}-1\right) P_{n}(0)$.

ii. If $n$ is even, $Q_{n}(\sqrt{3})=\frac{1}{4}\left(3^{n+1}+1\right) Q_{n}(0)$.

Proof. Note first that $\cos 3 t=\cos t(2 \cos 2 t-1)$, by the addition formula for cosine and the double-angle formulas for sine and cosine. Then

$$
P(\sqrt{3}, t)=\frac{\sin t+\sqrt{3} \cos t}{\cos t-\sqrt{3} \sin t}=\frac{\sqrt{3}+2 \sin 2 t}{2 \cos 2 t-1}=\frac{(\sqrt{3}+2 \sin 2 t) \cos t}{\cos 3 t}
$$

while on the other hand

$$
\begin{aligned}
3 P(0,3 t)-P(0, t)=\frac{3(\sin 2 t \cos t+\sin t \cos 2 t)-(2 \cos 2 t-1) \sin t}{\cos 3 t} & \\
= & \frac{3 \sin 2 t \cos t+2 \cos ^{2} t \sin t}{\cos 3 t}=\frac{4 \sin 2 t \cos t}{\cos 3 t} .
\end{aligned}
$$

Thus

$$
P(\sqrt{3}, t)-\frac{1}{2}(3 P(0,3 t)-P(0, t))=\frac{\sqrt{3} \cos t}{\cos 3 t},
$$

and (i) follows from consideration of the coefficient of $t^{n} / n !, n$ odd (Note the right-hand side is an even function). A similar argument proves the identity

$$
Q(\sqrt{3}, t)-\frac{1}{4}(3 Q(0,3 t)+Q(0, t))=\frac{\sqrt{3}}{2} \frac{\sin 2 t}{\cos 3 t}
$$

from which (ii) follows upon the observation that the right-hand side is an odd function.

Theorem 3.3. $i$. If $n>0$ is even, $P_{n}(\sqrt{3})$ can be computed from the tangent numbers $P_{k}(0)$ via

$$
P_{n}(\sqrt{3})=\frac{\sqrt{3}}{2} \sum_{k \text { odd }}\left(\begin{array}{l}
n \\
k
\end{array}\right)\left(3^{k+1}-1\right) P_{k}(0) P_{n-k}(0)
$$

ii. If $n$ is odd, $Q_{n}(\sqrt{3})$ can be computed from the tangent numbers $P_{k}(0)$ and the secant numbers $Q_{k}(0)$ via

$$
Q_{n}(\sqrt{3})=\frac{\sqrt{3}}{8} \sum_{k \text { odd }}\left(\begin{array}{l}
n \\
k
\end{array}\right)\left(3^{k+1}-1\right) P_{k}(0) Q_{n-k}(0)
$$

Proof. For (i), set $s=\frac{\pi}{3}$ and $u=0$ in Theorem 2.2(i) to get

$$
P_{n}(\sqrt{3})-\sqrt{3} \sum_{k=0}^{n}\left(\begin{array}{l}
n \\
k
\end{array}\right) P_{k}(\sqrt{3}) P_{n-k}(0)=P_{n}(0)+\sqrt{3} \delta_{0 n}
$$


THE ElECtronic Journal of COMBinatorics 6 (1999),\#R21

Now suppose $n>0$ is even; then this reduces to

$$
P_{n}(\sqrt{3})=\sqrt{3} \sum_{k \text { odd }}\left(\begin{array}{l}
n \\
k
\end{array}\right) P_{k}(\sqrt{3}) P_{n-k}(0)=\sqrt{3} \sum_{k \text { odd }}\left(\begin{array}{l}
n \\
k
\end{array}\right) \frac{3^{k+1}-1}{2} P_{k}(0) P_{n-k}(0),
$$

where we have used Theorem 3.2(i) in the last step. For (ii), proceed similarly after setting $s=\frac{\pi}{3}$ and $u=-\sqrt{3}$ in Theorem $2.2(\mathrm{ii})$.

Remarks. 1. Comparing equation (6) to the equation at the beginning of $\S 24$ in [10], we see the numbers $H_{n}$ of $[9,10]$ are given by $H_{n}=\sqrt{3} P_{2 n}(\sqrt{3}) / 2^{2 n+1}$.

2. Similarly, equation (7) shows that the numbers $T_{n}$ of $[9]$ are $Q_{2 n-1}(\sqrt{3}) / \sqrt{3}$.

3. In Theorem 5.1 below we show that the numbers $P_{2 n}(\sqrt{3})$ and $Q_{2 n-1}(\sqrt{3})$ are closely related to certain generalized Euler and class numbers as defined in [17].

4. Root systems, values of derivative polynomials at $\mathbf{0}$ and $\mathbf{1}$, and the combinatorics of snakes. Let $V$ be a real vector space, $R$ a root system in $V$, and $W$ the Weyl group of $R$ (for definitions see [5]). Fix a set $S$ of simple roots for $R$ : then any $\alpha \in R$ is either a positive or negative linear combination of elements of $S$; we write $\alpha>0$ in the first case and $\alpha<0$ in the second. For $I \subset S$, denote by $\sigma(I, S)$ the number of elements $w \in W$ such that $w \alpha>0$ for $\alpha \in I$ and $w \alpha<0$ for $\alpha \in S-I$. Let $M(R)$ be the maximum value of $\sigma(I, S)$. T. A. Springer [19] computed the quantity $M(R)$ for all irreducible root systems $R$. Setting aside the exceptional root systems, his results are as follows.

1. If $R$ is of type $A_{n}, n \geq 1$, then $M(R)=a_{n}$ satisfies

$$
1+t+\sum_{n \geq 2} \frac{a_{n-1}}{n !} t^{n}=\tan t+\sec t
$$

2. If $R$ is of type $B_{n}$ or $C_{n}, n \geq 2$, then $M(R)=b_{n}$ satisfies

$$
1+t+\sum_{n \geq 2} \frac{b_{n}}{n !} t^{n}=\frac{\cos t+\sin t}{\cos 2 t}
$$

3. If $R$ is of type $D_{n}, n \geq 3$, then $M(R)=d_{n}$ satisfies

$$
t+\frac{1}{2} t^{2}+\sum_{n \geq 3} \frac{d_{n}}{n !} t^{n}=\frac{1+\sin 2 t-\cos t-\sin t}{\cos 2 t} .
$$

We shall call $M(R)$ the Springer number of the root system $R$.

Proposition 4.1. The Springer numbers $a_{n}, b_{n}$, and $d_{n}$ as defined above are given by $a_{n}=P_{n+1}(0)+Q_{n+1}(0), b_{n}=Q_{n}(1)$, and $d_{n}=P_{n}(1)-Q_{n}(1)$.

Proof. This amounts to writing the the generating functions above in terms of $P$ and $Q$. For example, the formula for $a_{n}$ follows from observing that $P(0, t)+Q(0, t)=\tan t+\sec t$. Similarly,

$$
Q(1, t)=\frac{1}{\cos t-\sin t}=\frac{\cos t+\sin t}{\cos 2 t}
$$


THE ELECTRONiC JOURNAL OF COMBINATORICS 6 (1999),\#R21

and

$$
P(1, t)-Q(1, t)=\frac{\sin t+\cos t-1}{\cos t-\sin t}=\frac{1+\sin 2 t-\cos t-\sin t}{\cos 2 t} .
$$

By describing Springer numbers geometrically in terms of Weyl chambers, Arnol'd [3] showed that the numbers $a_{n}, b_{n}$, and $d_{n}$ can be thought of as counting various types of snakes (updown sequences). The formal definitions are as follows.

Definition. A snake of type $A_{n}$ is a sequence $\left(x_{0}, x_{1}, \ldots, x_{n}\right)$ of integers such that $x_{0}<$ $x_{1}>x_{2}<\cdots x_{n}$ and $\left\{x_{0}, x_{1}, \ldots, x_{n}\right\}=\{0,1, \ldots, n\}$. A snake of type $B_{n}$ is a sequence $\left(x_{1}, x_{2}, \ldots, x_{n}\right)$ of integers such that $0<x_{1}>x_{2}<\cdots x_{n}$ and $\left\{\left|x_{1}\right|,\left|x_{2}\right|, \ldots,\left|x_{n}\right|\right\}=$ $\{1,2, \ldots, n\}$. A snake of type $D_{n}$ is a sequence $\left(x_{1}, \ldots, x_{n}\right)$ of integers such that $-x_{2}<$ $x_{1}<x_{2}>x_{3}<\cdots x_{n}$ and $\left\{\left|x_{1}\right|,\left|x_{2}\right|, \ldots,\left|x_{n}\right|\right\}=\{0,1, \ldots, n-1\}$.

We shall write $A_{n}$ for the set of snakes of type $A_{n}$ and so forth. The geometric argument of [3] shows that card $A_{n}$, card $B_{n}$, and card $D_{n}$ are $a_{n}, b_{n}$, and $d_{n}$ respectively. On the other hand, it is possible to prove that these cardinalities are given by the formulas of Proposition 4.1 using combinatorial arguments about snakes. This is done for $A_{n}$ in [2] and [3] (see the remark following Theorem 13): we do it here for $B_{n}$ and $D_{n}$. For this purpose, it is convenient to introduce another type of snake from [3]: an integer sequence $\left(x_{1}, \ldots, x_{n}\right)$ such that $x_{1}<x_{2}>x_{3}<\cdots x_{n}$ and $\left\{\left|x_{1}\right|, \ldots,\left|x_{n}\right|\right\}=\{1, \ldots, n\}$ is called a snake of type $\beta_{n}$. Let $\beta_{n}$ denote the set of snakes of type $\beta_{n}$.

Theorem 4.2. Let $b(t)=\sum_{n \geq 0} \operatorname{card} B_{n} t^{n} / n$ ! and $\beta(t)=\sum_{n \geq 0} \operatorname{card} \beta_{n} t^{n} / n$ !. Then $b(t)=Q(1, t)$ and $\beta(t)=P(1, t)\left(\right.$ so $\operatorname{card} B_{n}=Q_{n}(1)$ and $\left.\operatorname{card} \beta_{n}=P_{n}(1)\right)$.

Proof. We prove the formula for $\beta(t)$ first. Given $\left(x_{1}, \ldots, x_{n+1}\right) \in \beta_{n+1}$, let $r$ be the unique element of $\{0, \ldots, n\}$ with $\left|x_{r+1}\right|=n+1$. Then the sets $\left\{\left|x_{1}\right|, \ldots,\left|x_{r}\right|\right\}$ and $\left\{\left|x_{r+2}\right|, \ldots,\left|x_{n+1}\right|\right\}$ partition $\{1, \ldots, n\}$. The sequence $\left(x_{1}, \ldots, x_{r}\right)$ can be shrunk into a snake of type $\beta_{r}$ by applying the order-preserving bijection of $\left\{\left|x_{1}\right|, \ldots,\left|x_{r}\right|\right\}$ onto $\{1, \ldots, r\}$; similarly $\left((-1)^{r+1} x_{r+2}, \ldots,(-1)^{r+1} x_{n+1}\right)$ gives a snake of type $\beta_{n-r}$. Conversely, given $r \in\{0, \ldots, n\}$ and a partition of $\{1, \ldots, n\}$ into an $r$-set and an $(n-r)$-set, together with elements of $\beta_{r}$ and $\beta_{n-r}$, we can construct a $\beta_{n+1}$-snake in a unique way. Hence

$$
\operatorname{card} \beta_{n+1}=\sum_{r=0}^{n}\left(\begin{array}{l}
n \\
r
\end{array}\right) \operatorname{card} \beta_{r} \operatorname{card} \beta_{n-r}+\delta_{n 0} .
$$

from which follows $\beta^{\prime}(t)=\beta(t)^{2}+1$. (The Kronecker delta term reflects the fact that there are two $\beta_{1}$-snakes, $(1)$ and $(-1)$.) The unique solution of this differential equation satisfying the initial condition $\beta(0)=\operatorname{card} \beta_{0}=1$ ( $\beta_{0}$ consists of the empty snake) is

$$
\beta(t)=\tan \left(t+\frac{\pi}{4}\right)=\frac{\tan t+1}{1-\tan t}=P(1, t) .
$$

Now suppose $\left(x_{1}, \ldots, x_{n+1}\right) \in B_{n+1}$, with $r \in\{0, \ldots, n\}$ such that $\left|x_{r+1}\right|=n+1$. Again the sequences $\left(x_{1}, \ldots, x_{r}\right)$ and $\left(x_{r+2}, \ldots, x_{n+1}\right)$ consist of integers whose absolute values partition $\{1, \ldots, n\}$. The sequence $\left(x_{1}, \ldots, x_{r}\right)$ can be shrunk into a $B_{r}$-snake, since 
THE ElECtronic Journal of COMBinatorics 6 (1999),\#R21

$x_{1}>0$; but the shrinkage of the sequence $\left((-1)^{r} x_{r+2}, \ldots,(-1)^{r} x_{n+1}\right)$ is a snake of type $\beta_{n-r}$. Hence

$$
\operatorname{card} B_{n+1}=\sum_{r=0}^{n}\left(\begin{array}{l}
n \\
r
\end{array}\right) \operatorname{card} B_{r} \operatorname{card} \beta_{n-r}
$$

and we have $b^{\prime}(t)=b(t) \beta(t)$. Using $b(0)=1$ and our formula for $\beta(t)$, this gives

$$
b(t)=\frac{1}{\sqrt{2}} \sec \left(t+\frac{\pi}{4}\right)=Q(1, t) .
$$

Theorem 4.3. $\operatorname{card} \beta_{n}=\operatorname{card} B_{n}+\operatorname{card} D_{n}\left(\right.$ so $\left.\operatorname{card} D_{n}=P_{n}(1)-Q_{n}(1)\right)$.

Proof. First note that we have a partition $\beta_{n}=\beta_{n}^{-} \cup \beta_{n}^{+}$, where $\beta_{n}^{-}$and $\beta_{n}^{+}$are respectively the sets of $\beta_{n}$-snakes that start with a negative integer and with a positive integer. We shall define bijections $f: \beta_{n}^{-} \rightarrow B_{n}$ and $g: \beta_{n}^{+} \rightarrow D_{n}$. Let $f\left(x_{1}, \ldots, x_{n}\right)=\left(-x_{1}, \ldots,-x_{n}\right)$ : it is easy to see that $f$ is a bijection of $\beta_{n}^{-}$onto $B_{n}$. For $g$, let $\left(x_{1}, \ldots, x_{n}\right) \in \beta_{n}^{+}$, with $r \in\{1, \ldots, n\}$ such that $\left|x_{r}\right|=1$. Then $g\left(x_{1}, \ldots, x_{n}\right)=\left(x_{r} \tilde{x}_{1}, \tilde{x}_{2}, \ldots, \tilde{x}_{n}\right)$, where $\tilde{x}_{i}=\left(\operatorname{sgn} x_{i}\right)\left(\left|x_{i}\right|-1\right)$. The reader may verify that the image of $g$ is in $D_{n}$, and in fact that $g$ has an inverse given by

$$
g^{-1}\left(y_{1}, \ldots, y_{n}\right)= \begin{cases}\left(1, \hat{y}_{2}, \ldots, \hat{y}_{n}\right), & \text { if } r=1 \\ \left(\left|\hat{y}_{1}\right|, \hat{y}_{2}, \ldots, \hat{y}_{r-1}, \operatorname{sgn} y_{1}, \hat{y}_{r+1}, \ldots, \hat{y}_{n}\right), & \text { otherwise }\end{cases}
$$

for $\left(y_{1}, \ldots, y_{n}\right) \in D_{n}$ with $y_{r}=0$, and $\hat{y}_{i}=\left(\operatorname{sgn} y_{i}\right)\left(\left|y_{i}\right|+1\right)$ for $i \neq r$.

We can use the machinery of previous sections to obtain relations among the Springer numbers (and card $\beta_{n}$ ). For example, equation (5) above implies card $\beta_{n}=2^{n} a_{n-1}$, which has a simple combinatorial interpretation in terms of snakes (cf. Theorem 24 of [3]). Other relations, like the following, appear to be new.

Proposition 4.4. For positive integers $n$,

$$
b_{n}=\frac{(-1)^{n}+1}{2} a_{n-1}+\sum_{k \text { odd }}\left(\begin{array}{l}
n \\
k
\end{array}\right) a_{k-1} b_{n-k}
$$

and

$$
d_{n}=(-1)^{n-1} a_{n-1}+\sum_{k \text { odd }}\left(\begin{array}{l}
n \\
k
\end{array}\right) a_{k-1} d_{n-k}
$$

Proof. Set $s=\frac{\pi}{4}$ and $u=0$ in Theorem 2.2: then the first identity follows from part (ii) of the theorem, and the second upon subtracting part (ii) from part (i).

Remark. The formula for $b_{n}$ can be given a combinatorial interpretation. Let $\bar{A}_{m}$ be the set of sequences $\left(x_{0}, \ldots, x_{m}\right)$ such that $\left\{x_{0}, \ldots, x_{m}\right\}=\{0,1, \ldots, m\}$ and $x_{0}>x_{1}<x_{2}>$ $\cdots x_{m}$ : evidently $\bar{A}_{m}$ is in 1-1 correspondence with $A_{m}$ via $\left(x_{0}, \ldots, x_{m}\right) \rightarrow\left(m-x_{0}, \ldots, m-\right.$ $\left.x_{m}\right)$. Now suppose $\left(x_{1}, \ldots, x_{n}\right) \in B_{n}$. If all the $x_{i}$ are positive, then $\left(x_{1}-1, \ldots, x_{n}-1\right) \in$ $\bar{A}_{n-1}$. Otherwise, there is a smallest $k \in\{1, \ldots, n\}$ with $x_{k}<0$, and it follows from the 
THE ELECTRONiC JOURNAL OF COMBINATORICS 6 (1999),\#R21

definition of $B_{n}$ that $k$ must be even. Then $\left(x_{1}, \ldots, x_{k-1}\right)$ can be shrunk into an element of $\bar{A}_{k-2}$, and $\left(-x_{k},-x_{k+1}, \ldots,-x_{n}\right)$ can be shrunk into an element of $B_{n-k+1}$. Since there are $\left(\begin{array}{c}n \\ k-1\end{array}\right)$ ways to choose $\left\{x_{1}, \ldots, x_{k-1}\right\} \subset\{1, \ldots, n\}$, we have

$$
b_{n}=a_{n-1}+\sum_{2 \leq k \leq n \text { even }}\left(\begin{array}{c}
n \\
k-1
\end{array}\right) a_{k-2} b_{n-k+1}=a_{n-1}+\sum_{k \leq n-1 \text { odd }}\left(\begin{array}{l}
n \\
k
\end{array}\right) a_{k-1} b_{n-k},
$$

which is equivalent to the first identity above.

5. Values of derivative polynomials at $\sqrt{3}$, generalized Euler and class numbers, and 3-signed permutations. In [17] Shanks defined positive integers $c_{a, n}$ (for integer $a \geq 1$ and $n \geq 0$ ) and $d_{a, n}$ (for integer $a, n \geq 1$ ) by

$$
L_{a}(2 n+1)=K_{a} \sqrt{a} \frac{c_{a, n}}{(2 n) !}\left(\frac{\pi}{2 a}\right)^{2 n+1} \quad \text { and } \quad L_{-a}(2 n)=K_{a} \sqrt{a} \frac{d_{a, n}}{(2 n-1) !}\left(\frac{\pi}{2 a}\right)^{2 n}
$$

where $K_{a}=\frac{1}{2}$ if $a=1$ and 1 otherwise, and

$$
L_{a}(s)=\sum_{k=0}^{\infty}\left(\frac{-a}{2 k+1}\right)(2 k+1)^{-s}
$$

here $(-a /(2 k+1))$ is the Jacobi symbol. As noted in [17], the numbers $c_{1, n}$ are just the secant numbers $Q_{2 n}(0)$, and the $d_{1, n}$ are the tangent numbers $P_{2 n-1}(0)$. Comparison of the tables in [17] and those of [3] reveals that the numbers $c_{2, n}$ and $d_{2, n}$ are Springer numbers: in fact $c_{2, n}=Q_{2 n}(1)=b_{2 n}$ and $d_{2, n}=Q_{2 n-1}(1)=b_{2 n-1}$. This can be proved using the recurrences given in [17] together with the generating function for the $Q_{n}(1)$ : see Proposition 6.3 of [16], where $b_{n}$ is denoted $E_{n}^{ \pm}$. Our next result gives the third row of Shanks's numbers in terms of the numbers $P_{2 n}(\sqrt{3})$ and $Q_{2 n-1}(\sqrt{3})$ discussed in Theorem 3.3 above.

Theorem 5.1. i. For $n \geq 0, c_{3, n}=\frac{1}{\sqrt{3}} P_{2 n}(\sqrt{3})$.

ii. For $n \geq 1, d_{3, n}=\frac{2}{\sqrt{3}} Q_{2 n-1}(\sqrt{3})$.

Proof. By substituting into the first of equations (19) of [17] the constants corresponding to the expression for $L_{3}(s)$ in equations (19) of [18], we have

$$
\sum_{n=0}^{\infty} w^{2 n} \frac{c_{3, n}}{(2 n) !}=\frac{\cos (3 w(1-4 / 3))}{\cos 3 w}=\frac{\cos w}{\cos 3 w}
$$

and comparison with equation (6) above proves (i). Similarly, substitute into the second of equations (19) of [17] the constants from the expression for $L_{-3}(s)$ in equations (19) of [18] to get

$$
\sum_{n=1}^{\infty} w^{2 n-1} \frac{d_{3, n}}{(2 n-1) !}=\frac{\sin (3 w(1-4 / 12))}{\cos 3 w}=\frac{\sin 2 w}{\cos 3 w}
$$


which on comparison with equation (7) gives (ii).

The notion of an alternating permutation of $\{1,2, \ldots, n\}$ is generalized in [8] to a " $\Lambda$ alternating augmented $r$-signed permutation" of $\{1,2, \ldots, n\}$ for any pair $(p, r)$ of positive integers with $p \leq r$. The cases $(1,1),(1,2)$ and $(2,2)$ correspond respectively to the $A_{n-1}$-snakes, $B_{n}$-snakes, and $\beta_{n}$-snakes of the previous section. Here we give a combinatorial interpretation of the numbers $c_{3, n}$ and $d_{3, n}$ using the case $(p, r)=(2,3)$. Let $S=\left\{n \omega^{m} \mid n, m\right.$ nonnegative integers $\}$, where $\omega=e^{\frac{2 \pi i}{3}}$, with the linear order

$$
\omega^{2}<2 \omega^{2}<3 \omega^{2}<\cdots<0<\omega<2 \omega<3 \omega<\cdots<1<2<3<\cdots
$$

Define an $E R_{n}$-snake to be a sequence $\left(x_{1}, x_{2}, \ldots, x_{n}\right)$ of elements of $S$ such that $0<x_{1}>$ $x_{2}<\cdots x_{n}$ and $\left\{\left|x_{1}\right|,\left|x_{2}\right|, \ldots,\left|x_{n}\right|\right\}=\{1,2, \ldots, n\}$. Let $E R_{n}$ be the set of $E R_{n}$-snakes, so e.g., $E R_{0}$ consists of the empty snake, $E R_{1}=\{(1),(\omega)\}$, and

$$
E R_{2}=\left\{(2,1),(2, \omega),\left(2, \omega^{2}\right),(2 \omega, \omega),\left(2 \omega, \omega^{2}\right),(1,2 \omega),\left(1,2 \omega^{2}\right),\left(\omega, 2 \omega^{2}\right)\right\} .
$$

Theorem 5.2. For $n \geq 0, c_{3, n}=\operatorname{card} E R_{2 n}$; for $n \geq 1, d_{3, n}=\operatorname{card} E R_{2 n-1}$.

Proof. In the terminology of [8], $E R_{n}$-snakes are $\Lambda$-alternating augmented 3 -signed permutations of $\{1,2, \ldots, n\}$ corresponding to $p=2$. By Proposition 7.2 of [8], we have

$$
\sum_{n=0}^{\infty} \frac{\operatorname{card} E R_{n}}{n !} x^{n}=\frac{\sin 2 x+\cos x}{\cos 3 x}
$$

and the conclusion follows by comparison with equations (8) and (9) above.

6. Euler Polynomials. In this section we give explicit formulas for the values of the Euler polynomials at rational numbers in terms of the $P_{n}$ and $Q_{n}$. The Euler polynomials $E_{n}(x)$ are defined by equation (2) above; the Euler numbers are $E_{n}=2^{n} E_{n}\left(\frac{1}{2}\right)$. In view of the translation formula for Euler polynomials (23.1.7 of [1]), it suffices to give formulas for rational arguments between 0 and 1 .

Theorem 6.1. If $n, p$ and $q$ are nonnegative integers with $0 \leq p \leq q(0<p<q$ if $n=0)$ and $q \geq 2$ even, then

$$
E_{n}\left(\frac{p}{q}\right)=\frac{2}{q^{n+1}} \sum_{k=0}^{q / 2-1} \sin \left(\frac{(2 k+1) \pi p}{q}-\frac{n \pi}{2}\right) \csc \frac{(2 k+1) \pi}{q} Q_{n}\left(\cot \frac{(2 k+1) \pi}{q}\right)
$$

if $p$ is odd, and

$$
E_{n}\left(\frac{p}{q}\right)=\frac{2}{q^{n+1}} \sum_{k=0}^{q / 2-1} \sin \left(\frac{(2 k+1) \pi p}{q}-\frac{n \pi}{2}\right) P_{n}\left(\cot \frac{(2 k+1) \pi}{q}\right)
$$

if $p$ is even.

Proof. We start with the Fourier series

$$
E_{n}\left(\frac{p}{q}\right)=\frac{4 \cdot n !}{\pi^{n+1}} \sum_{k=0}^{\infty} \frac{\sin \left(\frac{(2 k+1) \pi p}{q}-\frac{n \pi}{2}\right)}{(2 k+1)^{n+1}}
$$


THE ELECTRONiC JOURNAL OF COMBINATORICS 6 (1999),\#R21

([1], 23.1.16). Consider

$$
\psi(j)= \begin{cases}\sin \left(\frac{j \pi p}{q}-\frac{n \pi}{2}\right), & j \text { odd }, \\ 0, & j \text { even. }\end{cases}
$$

Then $\psi(j+q)=(-1)^{p} \psi(j)$, so $\psi$ is alternating $\bmod q$ if $p$ is odd and periodic $\bmod q$ if $p$ is even. Further, $\psi(q-j)=(-1)^{p+n+1} \psi(j)$, so $\psi$ has parity (as an alternating or periodic function $\bmod q$ ) opposite that of $p+n$. Thus, since

$$
E_{n}\left(\frac{p}{q}\right)=\frac{4 \cdot n !}{\pi^{n+1}} \sum_{j=1}^{\infty} \frac{\psi(j)}{j^{n+1}}
$$

the conclusion follows from Theorem 2.3.

Remark. By a proof similar to that above, one can obtain a formula for rational values $B_{n}(p / q)$ of the $n$th Bernoulli polynomial in terms of $P_{n-1}$. This is essentially Theorem C of $[20]$.

Examples. Set $q=2$ to get

$$
E_{n}\left(\frac{1}{2}\right)=\frac{1}{2^{n}} \sin \frac{(1-n) \pi}{2} Q_{n}(0)= \begin{cases}0, & n \text { odd } \\ (-1)^{\frac{n}{2}} 2^{-n} Q_{n}(0), & n \text { even. }\end{cases}
$$

Hence (by Theorem 2.1) $Q_{n}(0)=\left|E_{n}\right|$. We have also

$$
E_{n}(0)=-E_{n}(1)=\frac{1}{2^{n}} \sin \frac{(-n) \pi}{2} P_{n}(0)= \begin{cases}0, & n \text { even } \\ (-1)^{\frac{n+1}{2}} 2^{-n} P_{n}(0), & n \text { odd },\end{cases}
$$

for $n \geq 1$; this can be written in terms of Bernoulli numbers (see [1], 23.1.20) as $-2\left(2^{n+1}-\right.$ 1) $B_{n+1} /(n+1)$.

Taking $q=4$, we obtain

$$
E_{n}\left(\frac{1}{4}\right)=(-1)^{n} E_{n}\left(\frac{3}{4}\right)= \begin{cases}(-1)^{\frac{n}{2}} 4^{-n} Q_{n}(1), & n \text { even } \\ (-1)^{\frac{n+1}{2}} 4^{-n} Q_{n}(1), & n \text { odd }\end{cases}
$$

Finally, let $q=6$ to get

$$
E_{n}\left(\frac{1}{6}\right)=(-1)^{n} E_{n}\left(\frac{5}{6}\right)= \begin{cases}2(-1)^{\frac{n}{2}} 6^{-n-1}\left[2 Q_{n}(\sqrt{3})+Q_{n}(0)\right], & n \text { even, } \\ 4(-1)^{\frac{n+1}{2}} 6^{-n-1} \sqrt{3} Q_{n}(\sqrt{3}), & n \text { odd },\end{cases}
$$

and

$$
E_{n}\left(\frac{1}{3}\right)=(-1)^{n} E_{n}\left(\frac{2}{3}\right)= \begin{cases}2(-1)^{\frac{n}{2}} 6^{-n-1} \sqrt{3} P_{n}(\sqrt{3}), & n \text { even, } \\ 2(-1)^{\frac{n+1}{2}} 6^{-n-1}\left[P_{n}(\sqrt{3})-P_{n}(0)\right], & n \text { odd }\end{cases}
$$

If $n$ is even, we can use Theorem 3.2(ii) and equation (13) to write

$$
E_{n}\left(\frac{1}{6}\right)=E_{n}\left(\frac{5}{6}\right)=\frac{(-1)^{\frac{n}{2}}\left(3^{n}+1\right)}{2 \cdot 6^{n}} Q_{n}(0)=\frac{3^{n}+1}{2 \cdot 6^{n}} E_{n}
$$


(cf. [15], Ch. 2, eqn. (46)). If $n$ is odd, we have

$$
E_{n}\left(\frac{1}{3}\right)=-E_{n}\left(\frac{2}{3}\right)=\frac{(-1)^{\frac{n+1}{2}}\left(3^{n}-1\right)}{2 \cdot 6^{n}} P_{n}(0)=-\frac{\left(3^{n}-1\right)\left(2^{n+1}-1\right)}{3^{n}(n+1)} B_{n+1}
$$

using Theorem 3.2(i) and equation (14) (cf. [15], Ch. 2, eqn. (45); [1], 23.1.22).

We close this section by expressing the Springer and Shanks numbers in terms of the Euler polynomials.

Theorem 6.2. For integers $n \geq 1$,

$$
\begin{aligned}
a_{n-1} & =2^{n}\left|E_{n}\left(\frac{(-1)^{n}+1}{4}\right)\right| ; \\
b_{n} & =4^{n}\left|E_{n}\left(\frac{1}{4}\right)\right| ; \\
d_{n} & =4^{n}\left|E_{n}\left(\frac{(-1)^{n}+1}{4}\right)-E_{n}\left(\frac{1}{4}\right)\right| ; \\
c_{3, n} & =6^{2 n}\left|E_{2 n}\left(\frac{1}{3}\right)\right| ; \\
d_{3, n} & =6^{2 n-1}\left|E_{2 n-1}\left(\frac{1}{6}\right)\right| .
\end{aligned}
$$

Proof. For (i), note first that $a_{n-1}$ is $Q_{n}(0)$ for $n$ even, and $P_{n}(0)$ for $n$ odd; then use equations (10) and (11). For (ii), use equation (12). For (iii), observe from equation (5) that $d_{n}$ is $2^{n} Q_{n}(0)-Q_{n}(1)$ for $n$ even, and $2^{n} P_{n}(0)-Q_{n}(1)$ for $n$ odd; then use equation (12) together with equations (10) and (11) respectively. For (iv) and (v), use Theorem 5.1 together with equations (13) and (14). 
THE ELECTRONIC JOURNAL OF COMBINATORICS 6 (1999),\#R21

\section{REFERENCES}

1. M. Abramowitz and I. A. Stegun, Handbook of Mathematical Functions, National Bureau of Standards, Washington, D.C., 1964.

2. D. André, Sur les permutations alternées, J. Math. Pures Appl. (3) 7 (1881), 167-184.

3. V. I. Arnol'd, The calculus of snakes and the combinatorics of Bernoulli, Euler, and Springer numbers of Coxeter groups, Uspekhi Mat. Nauk. 47 (1992), 3-45 (Russian); Russian Math. Surveys 47 (1992), $1-51$.

4. M. D. Atkinson, How to compute the series expansions of $\sec x$ and $\tan x$, Amer. Math. Monthly 93 (1986), 387-389.

5. N. Bourbaki, Groupes et algèbres de Lie, Chap. 4,5,6, Hermann, Paris, 1968.

6. D. Cvijović and J. Klinowski, New formulae for the Bernoulli and Euler polynomials at rational arguments, Proc. Amer. Math. Soc. 123 (1995), 1527-1535.

7. D. Dumont, Further triangles of Seidel-Arnold type and continued fractions related to Euler and Springer numbers, Adv. Appl. Math. 16 (1995), 275-296.

8. R. Ehrenborg and M. A. Readdy, Sheffer posets and r-signed permutations, Ann. Sci. Math. Québec 19 (1995), 173-196.

9. J. W. L. Glaisher, On the Bernoullian function, Quarterly J. of Pure and Appl. Math. 29 (1898), $1-168$.

10. J. W. L. Glaisher, On a set of coefficients analogous to the Eulerian numbers, Proc. London Math. Soc. 31 (1899), 216-235.

11. J. W. L. Glaisher, On the coefficients in the expansions of $\cos x / \cos 2 x$ and $\sin x / \sin 2 x$, Quarterly J. of Pure and Appl. Math. 45 (1914), 187-222.

12. M. E. Hoffman, Derivative polynomials for tangent and secant, Amer. Math. Monthly 102 (1995), 23-30.

13. D. E. Knuth and T. J. Buckholtz, Computation of Tangent, Euler, and Bernoulli numbers, Math. Comp. 21 (1967), 663-688.

14. J. Millar, N. J. A. Sloane and N. E. Young, A new operation on sequences: the boustrophedon transform, J. Combin. Theory Ser. A 76 (1996), 44-54.

15. N. E. Nörlund, Vorlesungen über Differenzenrechnung, Chelsea, New York, 1954.

16. M. Purtill, André permutations, lexicographic shellability and the cd-index of a convex polytope, Trans. Amer. Math. Soc. 338 (1993), 77-104.

17. D. Shanks, Generalized Euler and class numbers, Math. Comp. 21 (1967), 689-694.

18. D. Shanks and J. W. Wrench, Jr., The calculation of certain Dirichlet series, Math. Comp. 17 (1963), $136-154$.

19. T. A. Springer, Remarks on a combinatorial problem, Nieuw Arch. Wisk. (3) 19 (1971), 30-36.

20. K. Wang, Exponential sums of Lerch's zeta functions, Proc. Amer. Math. Soc. 95 (1985), 11-15. 